\title{
Valeur pastorale des parcours naturels en zone soudano-guinéenne et stratégie paysanne d'adaptation aux effets de leur invasion par Chromolaena odorata au Bénin
}

\author{
André B. ABOH ${ }^{1,4^{*}}$, Sévérin BABATOUNDE ${ }^{2}$, Madjidou OUMOROU ${ }^{3,4}$, \\ Marcel HOUINATO ${ }^{4}$ et Brice SINSIN ${ }^{4}$ \\ ${ }^{1}$ Laboratoire de Recherches Zootechnique, Vétérinaire et Halieutique (LRZVH), Centre de Recherches \\ Agricoles d'Agonkanmey (CRA-Agonkanmey), Institut National des Recherches Agricoles du Bénin \\ (INRAB), 06 BP 629 Cotonou, Bénin. \\ ${ }^{2}$ Laboratoire de Zootechnie, Faculté des Sciences Agronomiques, Université d'Abomey-Calavi \\ 01 BP 526 Cotonou, Bénin. \\ ${ }^{3}$ Département de Génie de l'Environnement, Ecole Polytechnique d'Abomey-Calavi,Université \\ d'Abomey-Calavi, 01 BP 2009 Cotonou, Bénin. \\ 4 Laboratoire d'Ecologie Appliquée, Faculté des sciences Agronomiques,Université d'Abomey-Calavi \\ 01 BP 526 Cotonou, Bénin. \\ *Auteur correspondant, E-mail: aboh.solex@gmail.com ; a2abohboya@yahoo.fr ; Tel :00 22997931422
}

\section{RESUME}

Cette étude sur les pâturages naturels envahis par Chromolaena odorata a été réalisée dans la zone de transition guinéo-congolaise/soudanaise du Bénin. L'objectif de l'étude est d'analyser les connaissances endogènes, les paramètres biologique et agronomique pouvant permettre de déterminer l'effet de l'invasion de C. odorata sur le potentiel pastoral. Les valeurs du coefficient de similitude de Jaccard $(<50 \%)$, montre que les pâturages aux différents stades d'invasion ne sont pas similaires. La richesse spécifique est plus élevée au niveau des pâturages aux stades 1 et 2 d'invasion. La phytomasse des graminées a varié de 0,37 à 5,62 t MS/ha. Elle est plus élevée dans les pâturages au stade 1 d'invasion. La valeur pastorale $(1,12$ à 56,9$)$ et la capacité de charge ( $(0,00$ à $0,83 \mathrm{~kg} \mathrm{MS} / \mathrm{ha})$ diminuent avec l'intensité d'invasion par $C$. odorata. Les éleveurs disposent des connaissances endogènes sur la biologie de $C$. odorata et adaptent des stratégies d'élevage. Les critères de choix des espaces à pâturer sont par ordre de classification: les graminées consommées sans hésitation, les graminées consommées occasionnellement, le volume et l'accessibilité aux ressources fourragères. Le niveau d'invasion peuvent servir d'indicateurs essentiels de la qualité fourragère des parcours envahis en l'absence de toute analyse bromatologique.

(C) 2012 International Formulae Group. All rights reserved.

Mots clés: Chromolaena odorata, invasion biologique, capacité de charge, connaissances endogènes, élevage extensif.

\section{INTRODUCTION}

L'alimentation du bétail tropical en élevage extensif, repose sur le pâturage naturel qui constitue la base et le plus souvent la totalité des ressources alimentaires (Rivière, 1991 ; Sinsin, 1993). Selon les estimations,

(C) 2012 International Formulae Group. All rights reserved. DOI : http://dx.doi.org/10.4314/ijbcs.v6i4.22 
plus de $90 \%$ de l'énergie consommée par les bovins proviennent des pâturages, mais $10 \%$ des pâturages sont utilisés d'une façon non rationnelle (Pagot, 1985). Le débat sociétal sur les productions des animaux domestiques induit des questions de recherche qui doivent désormais répondre à des enjeux multiples. Il s'agit de maintenir les fonctions productives, voire les augmenter, favoriser les services environnementaux et réduire les externalités négatives comme l'érosion de la biodiversité. Une des causes majeures de l'érosion de la biodiversité indigène est la prolifération des espèces exotiques envahissantes (IUCN, 2004). Ces invasions biologiques sont considérées comme une question agricultureenvironnementale de premier ordre. Les invasions des plantes envahissantes affectent pratiquement tous les écosystèmes sur terre, mais le degré avec laquelle les différentes régions et les biomes sont envahis, et la qualité d'information de différentes régions, varie considérablement (Foxcroft et al., 2010). Les impacts négatifs dus aux invasions des plantes envahissantes dans les savanes africaines, semblent actuellement être moins grave que dans d'autres régions (D'Antonio et Vitousek, 1992; Foxcroft et al., 2010). Cependant, les récentes études montrent que 48 espèces exotiques, potentiellement envahisseurs sont recensées dans les savanes de l'Afrique du sud (Henderson, 2007) et autres localités comme le Bénin (Aboh et al., 2008). Selon Henderson (2007), Chromolaena odorata occupe la deuxième position des espèces potentiellement envahissantes. Elle prolifère dans les parcours de la zone soudano-guinéenne du Bénin (Aboh et al., 2008). Malheureusement peu d'attention a été accordée jusqu'à présent à son invasion dans les parcours naturels de la zone d'étude. Ainsi, les données quantitatives sur le pâturage envahi, indispensables pour une gestion rationnelle des ressources pastorales disponibles et pour l'élaboration d'un plan d'aménagement sont insuffisantes. Cette étude met en exergue la problématique de maintien de la biodiversité locale et de sa productivité pour les animaux domestiques au cœur des questions de développement durable face à l'invasion de $C$. odorata dans les parcours naturels.

\section{MATERIEL ET METHODES Milieu d'étude}

L'étude est conduite au Bénin dans la région de Bétécoucou comprise entre $2^{\circ} 20^{\prime}$ et $2^{\circ} 28^{\prime}$ de longitude Est et $7^{\circ} 45^{\prime}$ et $7^{\circ} 52^{\prime}$ de latitude Nord. La température moyenne annuelle est de $27,4^{\circ} \mathrm{C}$. Elle chute à $20,5^{\circ} \mathrm{C}$ pendant l'harmattan. On distingue une longue saison de pluie de mi-mars à octobre avec un minimum de pluie en août et une saison sèche de novembre à mars. La pluviosité moyenne est de 1120,6 mm/an. Les principaux sols du site expérimental sont des luvisols ferriques $\mathrm{du}$ plateau qui sont sablo-limoneux, par endroits, fortement chargés de gravillons, de latérite ou de quartzite; les vertisols eutriques et les gleysols eutriques qui sont des sols hydromorphes de dépression (forment de terrains en creux) (FAO et al., 1999; Igué, 2000). L'emprise humaine a fortement marqué les formations végétales et plusieurs faciès sont plutôt anthropogènes. A chaque saison sèche, l'utilisation des feux comme moyen d'aménagement de ces parcours naturels était courante.

\section{Typologie des pâturages}

L'inventaire des pâturages a tenu compte de trois stades d'invasion, en fonction du degré d'invasion (Richardson et al., 2000; Oumorou et al., 2010) exprimé en pourcentage de recouvrement de sol de la plante envahissante au sein du pâturage naturel. Le stade 1 correspond au stade de contamination ou non envahie (Témoin); c'est le stade durant lequel la plante envahissante a un recouvrement variant de 0 à $10 \%$. Le stade 2 correspond au stade d'établissement de la plante envahissante, c'est-à-dire le début de 
l'invasion ; c'est le stade durant lequel la plante envahissante a un recouvrement variant de 10 à $40 \%$. Le stade 3 est atteint lorsque la plante envahissante est devenue abondante et dominante en présentant un recouvrement supérieur à $40 \%$. Les pâturages concernés sont présentés dans le Tableau 1. Les stades 2 et 3 correspondent au stade d'invasion. L'inventaire des pâturages est réalisé selon la méthode des relevés phytosociologiques (Braun-Blanquet, 1932). Un total de 24 relevés phytosociologiques a été effectué durant la période de maturation des espèces herbacées. L'aire considérée était de $20 \mathrm{~m}$ x $20 \mathrm{~m}$. Les fréquences relatives (FR) et les recouvrements moyens (RM) des espèces sont utilisés comme premiers critères de classement en vue d'établir la typologie des pâturages.

Le pourcentage d'espèces communes à deux types de pâturages par rapport aux espèces particulières à chacun des deux est exprimé par le coefficient de similitude ou coefficient de communauté de Jaccard (Sinsin, 1993) qui est utilisé pour comparer les différents types de pâturage.

L'expression mathématique de coefficient de Jaccard est : $1 \mathrm{j}=100 \times \mathrm{c} /(\mathrm{a}+\mathrm{b}-\mathrm{c})$

Avec $\mathrm{c}=$ nombre d'espèces communes aux pâturages $\mathrm{R} 1$ et $\mathrm{R} 2$, $\mathrm{a}=$ nombre d'espèces de $\mathrm{R} 1 ; \mathrm{b}=$ nombre d'espèces de R2.

$\mathrm{R} 1, \mathrm{R} 2$ = Numéro du relevé d'un type de pâturage

Selon cette méthode, il est convenu, dans le cadre de cette étude, que deux communautés végétales étaient similaires si $1 \mathrm{j}$ est supérieur ou égal à 50\%. Ce seuil de 50\% est admis dans les travaux phytosociologiques par plusieurs auteurs (Sinsin, 1993; Sokpon, 1995).

\section{Relevés linéaires et valeur pastorale}

Les relevés linéaires sont réalisés dans des placeaux de $20 \mathrm{~m}$ x $20 \mathrm{~m}$ installés dans chacun des pâturages identifiés. La méthode des points quadrats alignés de Daget et
Poissonet (1971) est utilisée. Elle a consisté à tendre dans les placeaux, un décamètre au dessus du toit du tapis herbacé. Une lecture verticale est faite tous les $20 \mathrm{~cm}$ le long de ce décamètre, à l'aide d'une tige de $1,5 \mathrm{~m}$ de long. Le nombre de points contacts total était égal à 100. A chaque point de lecture, tous les contacts avec feuilles ou chaumes et autres organes sont pris en compte, mais l'espèce est notée une seule fois par point de contact. La méthode des points quadrats permet d'établir la fréquence relative $\mathrm{FRi}$, la contribution spécifique de contact $\mathrm{CSCi}$, la fréquence spécifique Fsi. Les données de relevés linéaires sont exploitées pour calculer la valeur pastorale (VP) (Daget et Poissonet, 1971). VP $=0,25 \times \Sigma$ CSCi X Isi où Is $=$ Indice de qualité spécifique choisi sur l'échelle de 0 à 4 et $\mathrm{CSC}=$ Contribution Spécifique de Contact.

L'embroussaillement exprime le niveau de dégradation d'un pâturage et est représenté par la contribution spécifique des refus. La grille d'indice de qualité spécifique choisie fait appel à la notion d'acceptabilité car certaines espèces sont plus ou moins recherchées (Sinsin, 1993). La valeur des indices de qualité des espèces a tenu compte des valeurs utilisées par Sinsin (1993) et complétées sur le terrain pour les autres espèces à travers le suivi régulier aux pâturages de l'appétence de ces herbacées par les bovins en utilisant la grille de qualité spécifique adoptée par Sinsin (1993).

\section{Récolte de phytomasse et capacité de charge}

L'estimation de la phytomasse produite en période active est réalisée par la méthode de coupes rases au pic de biomasse. Trois placeaux de coupe de $10 \mathrm{~m}$ x $10 \mathrm{~m}$ sont installés dans chacun des pâturages étudiés. A l'intérieur de chaque placeau, sept placettes de $1 \mathrm{~m}^{2}$ sont choisies au hasard et les espèces sont coupées à l'aide des sécateurs (Sinsin, 1993). Les espèces sont triées en catégorie de 
graminées bonnes, moyennes et médiocres, légumineuses et divers fourrages. Des échantillons de $150 \mathrm{~g}$ par catégorie d'espèce sont pris et séchés à l'étuve à $105^{\circ} \mathrm{C}$ jusqu'à poids constant pour l'estimation de la production totale de biomasse en matière sèche. Les données de la phytomasse totale consommable sont utilisées pour calculer la capacité de charge annuelle de chaque pâturage (Agonyissa et Sinsin, 1998 ; Oumorou et al., 2010).

$\mathrm{CC}(\mathrm{UBT} / \mathrm{ha})=\frac{\mathrm{ki} \times \text { quantité de biomasse totale }}{6,25 \mathrm{~kg} \mathrm{MS} / \mathrm{UBT} / \mathrm{j} \times 365}$

DTE $($ UBT $/$ ha $)=\frac{1}{\text { CC annuelle }(\text { UBT / ha })}$ avec :

CC : Capacité de charge en UBT/ha ; Quantité de biomasse totale (kg de MS/ha) ; DTE : Demande en terre équivalente ; ki exprime que la biomasse potentielle est consommée à $1 / 3$ sans dénudation complète du pâturage où ; MS = matière sèche $;$ UBT $=$ unité de bétail tropical.

\section{Connaissances paysannes de l'invasion des pâturages naturels \\ Pour comprendre et apprécier la} connaissance paysanne de l'invasion de $C$. odorata dans les pâturages, les informations sont recueillies auprès des acteurs au cours des réunions de groupe (focus group). Le choix des participants à la réunion de groupe est fondé sur l'âge (25 à 50 ans), la catégorie socioprofessionnelle (34 éleveurs et 20 agriculteurs). Au total, un effectif de 54 participants appartenant aux différents groupes socioculturels (Peulh, Datcha et Fon) est retenu. Pour une meilleure gestion des réunions et afin d'obtenir le maximum d'informations, un effectif de 3 à 5 participants est retenu à chaque réunion de groupe. Au cours de la réunion, la technique de la maïeutique est utilisée. Cette technique est basée sur une interview au cours de laquelle de petites questions, sur la base d'un guide d'entretien, sont posées en vue d'obtenir une réponse plus précise. Les informations sont recueillies sur les critères de choix d'un espace à pâturer. La méthode de classification par pair est utilisée pour classer ces critères de choix d'un pâturage. Les autres aspects abordés étaient : les causes de la prolifération et l'évolution de la plante exotique depuis les 10 dernières années, la connaissance sur sa biologie, l'importance de cette plante exotique pour les activités agricoles et pastorales, les moyens de contrôle de plante et les diverses utilisations traditionnelles. Des observations de terrain sont réalisées pour une triangulation des informations recueillies. Ainsi, les différents troupeaux de bovins sont suivis aux pâturages au cours de deux périodes. Au cours de la première période, deux suivis sont effectués au mois de novembre avant les feux de végétation et le second, au mois de février après le passage de feux. Pour la seconde période, deux suivis sont également effectués en avril après la pluie précoce et en juillet en pleine saison humide. Chaque suivi a duré 5 jours. Les temps d'exploitation par pâturage sont enregistrés et les espèces consommées par les bovins sont aussi enregistrées.

\section{Analyse statistique}

L'analyse de variance est effectuée sur les données de valeur pastorale, de phytomasse et de capacité de charge de l'ensemble des pâturages avec le logiciel Statistica 6.0 (1998). Le test de Newmankeuls est utilisé pour séparer les groupes homogènes. Au niveau des sols du plateau, l'analyse de variance est réalisée pour comparer les données du sol de l'ensemble des pâturages non envahis (stade 1 d'invasion) et du sol de l'ensemble des pâturages envahis (stades 2 et 3 d'invasion).

\section{RESULTATS}

\section{Typologie des pâturages et richesse spécifique}

Les valeurs du coefficient de similitude de Jaccard $(\mathrm{Ij})$, pour les différents types de 
pâturages comparés deux à deux sont toutes inférieures à $50 \%$ (Tableau 1).Les listes floristiques et l'abondance des espèces dominantes ont montré huit types de pâturages :- F (+) : Le pâturage des savanes arbustivesarborées du plateau à Heteropogon contortus et Chromolaena odorata au stade 2 d'invasion ;

- G (+) : Le pâturage des savanes arbustivesarborées du plateau à Andropogon tectorum et Chromolaena odorata au stade 2 d'invasion ;

- H (+ +): Le pâturage à Chromolaena odorata des jachères au stade 3 d'invasion des plateaux.

- A (-) : Le pâturage des savanes arbustivesarborées des dépressions à Andropogon schirensis et Elymandra androphila au stade 1 d'invasion ;

- B (-) : Le pâturage des savanes herbeuses des dépressions à Brachiaria jubata et Desmodium hirtum au stade 1 d'invasion ;

- C (-) : Le pâturage des savanes arbustivesarborées du plateau à Andropogon tectorum et Anogeissus leiocarpa au stade 1 d'invasion;

- D (-) : Le pâturage des savanes arbustivesarborées du plateau à Heteropogon contortus et Pseudocedrela kotschyi au stade 1 d'invasion ; E (-) : Le pâturage des savanes arbustives-arborées du plateau à Hyparrhenia involucrata et Combretum collinum au stade 1 d'invasion ; La richesse spécifique est élevée au niveau des pâturages aux stades 1 et 2 d'invasion, mais faible au niveau des pâturages au stade 3 d'invasion.

\section{Effet de l'invasion sur la valeur pastorale}

La contribution spécifique de contact des différentes catégories fourragères et les valeurs pastorales moyennes sont présentées dans le Tableau 2. Les contributions des graminées bonnes et des graminées moyennes sont plus élevées dans les pâturages au stade 1 d'invasion à $A$. schirensis et $E$. androphila, à $B$. jubata et $D$. hirtum, à $H$. contortus et $P$. kotschyi et à A. tectorum et A. leiocarpa. Dans ces pâturages au stade 1 d'invasion, la contribution, des graminées bonnes a varié de 33 à $38 \%$ et celle des graminées moyennes de 15 à $59 \%$. S'agissant des pâturages au stade 2 d'invasion à A. tectorum et $C$. odorata et à $H$. contortus et $C$. odorata, la contribution de bonnes graminées a varié de 0 à $9,1 \%$ et celle de graminées moyennes de 15 à $21 \%$. La contribution de ces catégories fourragères est très faible dans les autres pâturages au stade 3 d'invasion. En général, les contributions de graminées bonnes et des graminées moyennes diminuent avec l'intensité de l'invasion.

Concernant les valeurs pastorales, elles sont plus élevées dans les pâturages au stade 1 d'invasion à A. schirensis et E. androphila $(\mathrm{VP}=56,9)$, à $B$. jubata et $D$. hirtum $(\mathrm{VP}=$ 51,0) ; suivis des pâturages au stade 1 d'invasion à A. tectorum et A. leiocarpa $(\mathrm{VP}=$ $44,1)$ et à $H$. contortus et $P$. kotschyi $(\mathrm{VP}=$ $43,5)$. Les valeurs pastorales sont moyennes $(17,7$ à 27,5) dans les pâturages au stade 2 d'invasion (A. tectorum et $C$. odorata et à $H$. contortus et $C$. odorata) Les valeurs pastorales sont plus faibles dans les autres types de pâturages au stade 3 d'invasion. Les contributions des refus des différents pâturages ont varié de $5 \%$ à $94 \%$. La plus forte contribution des refus a été enregistrée dans le pâturage au stade 3 d'invasion.

\section{Effet de l'invasion sur la production fourragère et la capacité de charge}

La phytomasse des bonnes graminées a varié de $352 \mathrm{~kg}$ MS/ha à $2125 \mathrm{~kg}$ MS/ha dans les pâturages au stade 1 d'invasion (Tableau 3). La phytomasse des graminées moyennes a varié de $2475 \mathrm{~kg} \mathrm{MS} / \mathrm{ha}$ à $3583 \mathrm{~kg} \mathrm{MS} / \mathrm{ha}$ dans les pâturages au stade 1 d'invasion. Les pâturages au stade 1 d'invasion à $H$. involucrata et $C$. collinum et à $A$. schirensis et E. androphila ont donné la plus forte productivité en phytomasses de graminées ( $\mathrm{P}$ $<0,05)$. Ils sont suivis des pâturages au stade 1 d'invasion à $A$. tectorum et $A$. leiocarpa, à $H$. contortus et $P$. kotschyi et à $B$. jubata et $D$. hirtum. La productivité en graminée est 
moyenne dans les pâturages au stade 2 d'invasion à $A$. tectorum et $C$. odorata et à $H$. contortus et $C$. odorata. Aussi, les capacités de charge les plus élevées $(0,53$ à 0,83 UBT/ha) $(\mathrm{P}<0,05)$ sont elles enregistrées dans les pâturages au stade 1 d'invasion (à $H$. involucrata et $C$. collinum, à $A$. schirensis et E. androphila, A. tectorum et A. leiocarpa, à $H$. contortus et $P$. kotschyi, et à $B$. jubata et $D$. hirtum). Les pâturages au stade 2 d'invasion (à $A$. tectorum et $C$. odorata et à $H$. contortus et $C$. odorata) ont présenté une capacité de charge moyenne $(0,06$ à $0,32 \mathrm{UBT} / \mathrm{ha})$. Les productivités faibles en phytomasses de graminées et en capacités de charge $(0,00$ $\mathrm{UBT} / \mathrm{ha}$ ) ont été enregistrées dans les pâturages au stade 3 d'invasion. Le poids des refus qui variait de 15 à $5306 \mathrm{~kg}$ MS/ha est plus élevé, dans le pâturage au stade 3 d'invasion suivi des pâturages au stade 2 d'invasion. La productivité en légumineuse était similaire quelque soit le niveau d'invasion $(\mathrm{P}>0,05)$ avec une tendance plus élevée sur le pâturage au stade 1 d'invasion à $H$. involucrata et $C$. collinum et le pâturage au stade 3 d'invasion. La productivité en divers fourrages est plus élevée au niveau du pâturage à $A$. tectorum et $C$. odorata au stade 2 d'invasion $(\mathrm{P}<0,05)$.

\section{Connaissances endogènes et exploitation des pâturages envahis}

Chromolaena

odorata est communément appelé en langue locale "fongbé" agatou (fusil de haut) ou sékou touré ma (la plante de Sékou Touré). Un effectif de $71 \%$ des éleveurs a estimé que l'espèce se développe dans les endroits moins perturbés. Les participants enquêtés ont témoigné unanimement que l'espèce n'est pas consommée par les herbivores même en cas de famine. Toutefois, elle améliore la fertilité du sol avec un rendement élevé de maïs après sa jachère. Le développement de l'espèce diminue l'herbage consommable sur les pâturages. L'ensemble des enquêtés a affirmé qu'au stade 3 d'invasion, l'espèce contrôle efficacement Imperata cylindrica, autres mauvaises herbes des cultures et même les espèces ayant une bonne valeur pastorale comme les hémicryptophytes. Les participants ont signalé que $C$. odorata est pérenne, son expansion augmente l'embroussaillement des pâturages. Selon les enquêtés, la plante a aussi des vertus médicinales multiples contre l'abcès, la plaie, la gale et pour des actions mystiques.

D'un point de vue biologique, les enquêtés ont indiqué que l'espèce se reproduisent par les graines dont la distribution est assurée par le vent, l'eau et parfois les animaux et les hommes. Le contrôle de $C$. odorata dans les champs agricoles est réalisé grâce à l'essouchage manuel avec une main d'œuvre qui coûte de plus en plus cher et n'est pas toujours disponible. Le plant arraché doit être brulé afin d'éviter sa reprise. Par ailleurs, le feu de végétation joue un rôle important pour le contrôle de l'espèce en rendant infertile les graines.

L'inventaire des critères du choix de l'espace à pâturer a révélé que les éleveurs accordent une priorité aux graminées consommées sans hésitation $(24,1 \%)$, suivi des graminées consommées occasionnellement (16,7\%). Les autres critères importants sont le volume des ressources fourragères $(14,8 \%)$, l'accessibilité aux ressources fourragères $(14,8 \%)$ et la présence des ligneux $(13,0 \%)$. Les autres paramètres inventoriés sont: les autres ressources fourragères $(7,4 \%)$, le stade végétatif $(7,4 \%)$ et le temps de repos des parcours $(1,9 \%)$.

La Figure 1 indique l'adaptation du cycle de pâture en fonction des saisons climatiques, des pratiques du feu de végétation et du cycle de reproduction de $C$. odorata. Les pâturages aux stades 1 et 2 d'invasion sont exploités en toute saison. 


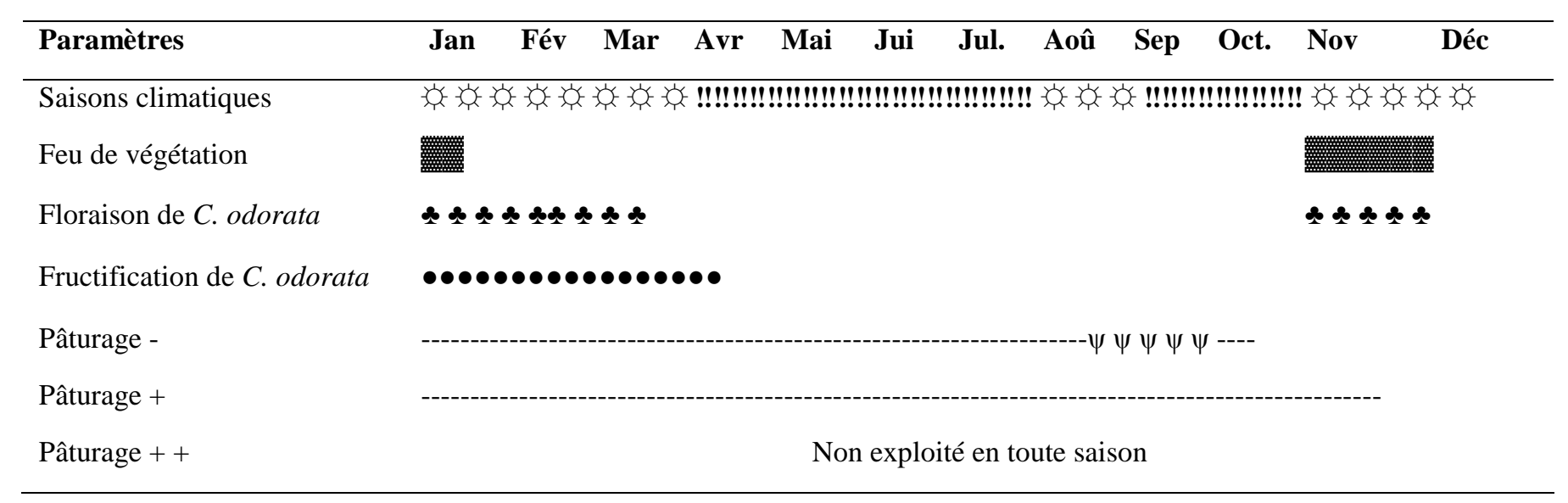

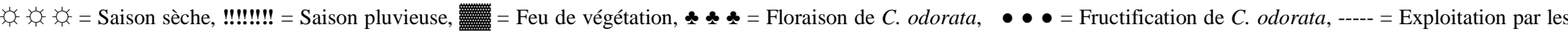
bovins, $\psi \psi \psi=$ Constitution de foin puis pâture par les bovins, Pâturage - = Pâturage au stade 1 d'invasion par $C$. odorata ; Pâturage $+=$ Pâturage au stade 2 d'invasion par $C$ odorata, Pâturage + + = Pâturage au stade 3 d'invasion par C. odorata

Figure 1: Exploitation des pâturages par les bovins en relation avec le niveau d'invasion par $C$. odorata, son cycle de reproduction, les saisons climatiques et les pratiques de feux de végétation. 
Tableau 1: Degré de communauté entre les différents types de pâturages comparés deux à deux.

\begin{tabular}{|c|c|c|c|c|c|c|c|c|c|c|c|}
\hline $\mathbf{P}$ & $\mathbf{R S}$ & $\mathbf{P}$ & RS & EC & $\mathbf{I j}(\%)$ & $\mathbf{P}$ & RS & $\mathbf{P}$ & $\mathbf{R S}$ & EC & $\mathbf{I j}(\%)$ \\
\hline A (-) & 32 & B (-) & 24 & 9 & 19,15 & C (-) & 62 & E (-) & 42 & 19 & 22,35 \\
\hline A (-) & 32 & $C(-)$ & 62 & 8 & 9,30 & $C(-)$ & 62 & $F(+)$ & 48 & 11 & 11,11 \\
\hline$A(-)$ & 32 & $D(-)$ & 70 & 11 & 12,09 & $C(-)$ & 62 & $\mathrm{G}(+)$ & 51 & 29 & 34,52 \\
\hline A (-) & 32 & E (-) & 42 & 13 & 21,31 & $C(-)$ & 62 & $\mathrm{H}(++)$ & 12 & 3 & 4,23 \\
\hline$A(-)$ & 32 & $F(+)$ & 48 & 8 & 11,11 & D (-) & 70 & E (-) & 42 & 28 & 33,33 \\
\hline$A(-)$ & 32 & $\mathrm{G}(+)$ & 51 & 11 & 15,28 & $\mathrm{D}(-)$ & 70 & $\mathrm{~F}(+)$ & 48 & 17 & 16,83 \\
\hline A (-) & 32 & $\mathrm{H}(++)$ & 12 & 2 & 4,76 & D (-) & 70 & $\mathrm{G}(+)$ & 51 & 20 & 19,80 \\
\hline B (-) & 24 & C (-) & 62 & 4 & 4,88 & D (-) & 70 & $\mathrm{H}(++)$ & 12 & 5 & 6,49 \\
\hline B (-) & 24 & D (-) & 70 & 9 & 10,59 & E (-) & 42 & $\mathrm{~F}(+)$ & 48 & 10 & 12,50 \\
\hline B (-) & 24 & E (-) & 42 & 4 & 6,45 & E (-) & 42 & $\mathrm{G}(+)$ & 51 & 18 & 24,00 \\
\hline B (-) & 24 & $\mathrm{~F}(+)$ & 48 & 3 & 4,35 & E (-) & 42 & $\mathrm{H}(++)$ & 12 & 3 & 5,88 \\
\hline B (-) & 24 & $G(+)$ & 51 & 3 & 4,17 & $F(+)$ & 48 & $\mathrm{G}(+)$ & 51 & 15 & 17,86 \\
\hline B (-) & 24 & $\mathrm{H}(++)$ & 12 & 1 & 2,86 & $\mathrm{~F}(+)$ & 48 & $\mathrm{H}(++)$ & 12 & 5 & 9,09 \\
\hline C (-) & 62 & D (-) & 70 & 28 & 26,92 & $\mathrm{G}(+)$ & 51 & $\mathrm{H}(++)$ & 12 & 4 & 6,78 \\
\hline
\end{tabular}

$\mathrm{P}=$ pâturage $; \mathrm{RS}=$ Richesse spécifique $; \mathrm{EC}=$ effectif d'espèces communes $; \mathrm{I}_{\mathrm{j}}=$ Coefficient de Jaccard ; A $(-)=$ Pâturage à $A$. schirensis et $E$. androphila au stade $1 \mathrm{~d}$ 'invasion ; B $(-)=$ Pâturage à $B$. jubata et $D$. hirtum au stade 1 d'invasion ; C (-) = Pâturage à A. tectorum et A. leiocarpa au stade 1 d'invasion ; D $(-)=$ Pâturage à $H$. contortus et $P$. kotschyi au stade 1 d'invasion; E (-) = Pâturage à $H$. involucrata et $C$. collinum au stade 1 d'invasion ; F (+) = Pâturage à $H$. contortus et $C$. odorata au stade 2 d'invasion ; $\mathrm{G}(+)=$ Pâturage à $A$. tectorum et $C$. odorata au stade 2 d'invasion ; $\mathrm{H}(++)=$ Pâturage à $C$. odorata au stade 3 d'invasion.

Par contre, les pâturages au stade 3 d'invasion sont abandonnés en toute saison à cause de leur inaccessibilité. Le feu de végétation précoce est allumé en novembre et décembre, ce qui engendre une réduction de l'herbage disponible. Les ligneux jouent un rôle important dans l'alimentation surtout en saison sèche et davantage avec l'arrivée des bovins transhumants surtout étrangers qui constituent une charge importante qui s'exerce sur les parcours naturels. Le concentré à base de tourteau de coton dont la composition chimique se présente comme suit : $34,6 \%$ de MAT; $12 \%$ de Ca ; 0,97\% de P sont distribués aux bovins de la ferme d'élevage de Bétécoucou. L'avènement des pluies précoces, en mars ou avril, marque la reprise des graminées pérennes.

\section{DISCUSSION}

\section{Invasion et valeur pastorale}

Cette étude met en exergue la problématique de l'invasion des plantes envahissantes telle que $C$. odorata dans les parcours naturel de la zone soudanoguinéenne. L'analyse floristique s'appuyant sur l'utilisation du coefficient de Jaccard montre que les pâturages aux différents stades d'invasion ne sont pas similaires. Les invasions biologiques changent évidemment la composition et la structure des communautés des plants, et également les écosystèmes (Witkowski et Wilson, 2001) comme le démontre la présente étude. Toutefois, cette altération de la flore du parcours ne saurait être attribuée seulement à l'invasion par $C$. odorata. Les évolutions de la flore et la végétation des parcours communautaires constatées à un moment donné sont la résultante de facteurs écologiques (sols, climat), des effets des troupeaux domestiques et d'une action combinée de plusieurs facteurs anthropiques. 
Tableau 2: Contribution spécifique des différentes catégories fourragères (\%) et valeur pastorale des pâturages étudiés.

\begin{tabular}{|c|c|c|c|c|c|c|c|}
\hline \multirow[t]{2}{*}{ Types de pâturage } & \multicolumn{6}{|c|}{ Catégories fourragères (\%) } & \multirow[b]{2}{*}{ Valeur } \\
\hline & BG & MG & GMd & $\mathbf{L g}$ & DF & Refus & \\
\hline Indice de qualité & 4 & 2 à 3 & 1 & 1 à 2 & 1 & 0 & pastorale \\
\hline A. schirensis et E. androphila (-) & $33,0 \mathrm{~b}$ & $43 b$ & $6,5 \mathrm{c}$ & $9,67 \mathrm{abc}$ & $2,0 \mathrm{~b}$ & $5,0 \mathrm{c}$ & $56,9 a^{1}$ \\
\hline B. jubata et D. hirtum (-) & $0,0 \mathrm{~d}$ & $58 \mathrm{a}$ & $9,7 \mathrm{c}$ & $25,4 \mathrm{ab}$ & $0,4 \mathrm{~b}$ & $06,1 \mathrm{c}$ & $51,0 \mathrm{a}$ \\
\hline A. tectorum et A. leiocarpa (-) & $38,1 \mathrm{a}$ & $15,4 \mathrm{c}$ & $15,4 b$ & $17,5 \mathrm{abc}$ & $15,1 \mathrm{a}$ & $13,9 \mathrm{c}$ & $44,1 \mathrm{a}$ \\
\hline H. contortus et $P$. kotschyi (-) & $0,0 \mathrm{~d}$ & $59 \mathrm{a}$ & $1,0 \mathrm{~d}$ & $20 \mathrm{abc}$ & $1,9 b$ & $17,2 \mathrm{c}$ & $43,5 \mathrm{a}$ \\
\hline H. involucrata et $C$. collinum (-) & $0,0 \mathrm{~d}$ & $0,0 \mathrm{~d}$ & $58,7 \mathrm{a}$ & $31,9 \mathrm{a}$ & $0,66 b$ & $08,7 \mathrm{c}$ & $18,1 \mathrm{~b}$ \\
\hline$H$. contortus et $C$. odorata $(+)$ & $0,0 \mathrm{~d}$ & $21 \mathrm{c}$ & $2,0 \mathrm{~d}$ & $15,1 \mathrm{abc}$ & $4,5 b$ & $57,4 \mathrm{~b}$ & $27,53 b$ \\
\hline A. tectorum et C. odorata $(+)$ & $9,1 \mathrm{c}$ & $15,4 \mathrm{c}$ & $3,4 d$ & $5,1 \mathrm{bc}$ & $2,0 \mathrm{~b}$ & $62,7 b$ & $17,77 b$ \\
\hline C. odorata $(++)$ & 0,0 & $1,98 \mathrm{~d}$ & $5,41 \mathrm{~d}$ & $2,86 \mathrm{c}$ & $0,0 \mathrm{c}$ & $94,2 \mathrm{a}$ & $1,12 \mathrm{c}$ \\
\hline
\end{tabular}


Tableau 3: Variation de la biomasse (kg MS /ha) des différentes catégories fourragères et capacité de charge (en UBT/ha) des pâturages.

\begin{tabular}{|c|c|c|c|c|c|c|c|}
\hline \multirow[t]{2}{*}{ Types de pâturage } & \multirow[b]{2}{*}{ BG } & \multirow[b]{2}{*}{ MG } & \multicolumn{4}{|c|}{ Catégories fourragères } & \multirow{2}{*}{$\begin{array}{c}\text { Capacité de } \\
\text { charge }\end{array}$} \\
\hline & & & GMd & Lg & DF & Refus & \\
\hline Indice de qualité & 4 & 2 à 3 & 1 & 1 à 2 & 1 & $\mathbf{0}$ & \\
\hline A. schirensis et E. androphila (-) & $352 b$ & $2475 c$ & $2885 b$ & $377 \mathrm{a}$ & $13 b$ & $157 \mathrm{c}$ & $0,83(1,20) \mathrm{a}^{1}$ \\
\hline B. jubata et D. hirtum (-) & 0 & $3583 b$ & $55 \mathrm{~d}$ & $107 \mathrm{a}$ & $98 b$ & $83 c$ & $0,53(1,9) \mathrm{c}$ \\
\hline A. tectorum et A. leiocarpa (-) & $2125 \mathrm{a}$ & $0 \mathrm{~d}$ & $2346 c$ & $85 \mathrm{a}$ & $54 b$ & $15 \mathrm{c}$ & $0,65(1,54) b$ \\
\hline H. contortus et $P$. kotschyi (-) & $0 \mathrm{c}$ & $4096 \mathrm{a}$ & Od & $325 a$ & $20 \mathrm{~b}$ & $65 \mathrm{c}$ & $0,60(1,7) b c$ \\
\hline H. involucrata et $C$. collinum (-) & $0 \mathrm{c}$ & Od & $5622 \mathrm{a}$ & $908 \mathrm{a}$ & $13 b$ & $48 \mathrm{c}$ & $0,82(1,22) \mathrm{a}$ \\
\hline$H$. contortus et $C$. odorata $(+)$ & $314 b$ & $2056 c$ & $117 d$ & $43 \mathrm{a}$ & $18 b$ & $2243 b$ & $0,32 \mathrm{~d}$ \\
\hline A. tectorum et C. odorata $(+)$ & $0 \mathrm{c}$ & $128 \mathrm{~d}$ & $0 \mathrm{~d}$ & $115 \mathrm{a}$ & $456 a$ & $1951 b$ & $0,06 \mathrm{e}$ \\
\hline C. odorata $(++)$ & $0 \mathrm{c}$ & $0 \mathrm{~d}$ & Od & $919 \mathrm{a}$ & $195 b$ & $5306 a$ & $0,00(100) * f$ \\
\hline
\end{tabular}


Au niveau des sols du plateau, le faible effectif d'espèces enregistré au stade 3 d'invasion dans les jachères, est lié aux modifications des conditions écologiques dues à l'envahissement du milieu par $C$. odorata. En effet, cette plante crée d'ombrage aux plantes annuelles qui sont héliophiles. Il y a donc une compétition pour la lumière et les éléments nutritifs. Une telle situation serait combinée avec le broutage excessif des espèces indigènes, qui crée un milieu perméable à l'espèce envahissante $C$. odorata, non consommées par les herbivores.

Les écosystèmes de savanes se caractérisent par une strate herbacée supérieure continue, composée essentiellement de graminées vivaces et parsemée plus ou moins densément d'arbres ou d'arbustes à port fréquemment tortueux (Seiny-Boukar, 2003). La valeur pastorale dépend, en premier lieu, des espèces présentes dont l'appétibilité conditionne la fréquentation et l'ingestion (Oumourou et al., 2010). Cette qualité pastorale est proportionnelle à la contribution des bonnes et moyennes graminées. Ces graminées pérennes subissent les effets négatifs de $C$. odorata avec une régression marquée au stade 3 d'invasion. Cette étude révèle que $C$. odorata est un des facteurs majeurs de dégradation des pâturages de la savane soudano-guinéenne comme signalé par Watkinson (2001) et Aboh et al. (2008). Les niveaux d'invasion déterminent les principaux gradients de valeur pastorale, de production fourragère et de capacité de charge. Ces paramètres d'évaluation de potentiel pastoral des parcours diminuent avec l'intensité d'invasion. Cette altération se caractérise par une diminution des graminées de qualité bonne et moyenne. Par contre, la masse et la proportion des espèces non consommées augmente avec l'intensité d'invasion.

\section{Stratégie paysanne d'exploitation des parcours envahis}

Les résultats issus des entretiens de groupe ont confirmé que l'invasion de $C$. odorata représente une entrave à la production des animaux à travers l'embroussaillement important des parcours et révèlent la difficulté d'accessibilité aux ressources fourragères. Les participants ont pu décrire quelques aspects de la biologie de la plante exotique. Ils ont évoqué quelques facteurs biotiques et abiotiques qui favorisent sa propagation (Radosevich et al., 2003) et les implications économiques pour les activités agropastorales. Les participants ont apprécié et argumenté l'impact positif plus subtil de l'invasion de $C$. odorata sur la fertilité du sol, les mauvaises herbes et le rendement de maïs. Nos résultats ainsi que ceux d'autres auteurs confirment cette perception des producteurs (De Foresta et Schwartz, 1991 ; Akobundu et Ekeleme, 1996). Ainsi, cette espèce semble ne pas être un problème majeur pour les cultures annuelles, mais plutôt pour l'élevage extensif.

Dans des conditions d'élevage extensif, les parcours sont ouverts au bétail toute l'année. Chez les éleveurs Peulh de la zone d'étude, plusieurs critères utilisés pour évaluer des parcours sont similaires à ceux utilisés en phytoécologie qui reposent sur la détermination de la valeur pastorale (Akpo et al., 2002). La détermination de la valeur pastorale combine la composition, la contribution et l'indice de qualité des espèces herbacées. Cet indice de qualité traduit l'intérêt zootechnique de chaque espèce herbacée pour une ou plusieurs espèces animales (Daget et Poissonet, 1971) et a concerné, dans le cadre de cette étude, les bovins. Chez les éleveurs Peulh, l'intérêt zootechnique recouvre la palatabilité (graminées consommées sans hésitation et graminées consommées occasionnellement) et 
la productivité (le volume de ressources fourragères) (Oumorou et al., 2010).

Face aux effets néfastes de $C$. odorata sur les parcours rapportés, les cycles d'exploitation des pâturages suivent la rationalité des éleveurs afin d'adapter le taux de charge animale aux disponibilités fourragères. Cette stratégie adaptative des éleveurs, combine la dynamique des parcours en liaison avec les saisons. La gestion par le feu, la pâture intense et l'abandon des parcours sans intérêt zootechnique sont aussi des pratiques communes à la région des savanes (Bodoni, 2003). En effet, le passage des feux précoces et tardifs pendant la floraison et la fructification de $C$. odorata (novembrefévrier) provoque l'avortement des fleurs et réduit par conséquent la quantité et la qualité de graines produites de l'ordre de $68 \%$ à 100\% (Aboh et al., 2008).

Toutefois, la présence de $C$. odorata avec un recouvrement faible dans les parcours, n'entrave pas leurs exploitations. La contribution des parcours aux stades 1 et 2 d'invasion est d'autant plus importante qu'elle assure, en saison de pluie et même en saison sèche une grande partie de l'alimentation des animaux. Mais, très peu d'éleveurs prennent en compte le temps de repos des parcours lors de la conduite des animaux. Il en découle un surpâturage avec, pour conséquence, l'envahissement (Diallo, 1997).

\section{Conclusion}

La prolifération de $C$. odorata dans les parcours constitue un problème pastoral majeur dans le milieu d'étude. Cette propagation induit des effets délétères sur les parcours. Au stade 3 d'invasion les parcours sont abandonnés. Les pratiques agropastorales utilisées combinent la biologie de l'espèce, l'adaptation à la dynamique végétale, le climat et le contrôle de l'invasion au moyen du feu de végétation. A cet effet, les résultats obtenus indiquent qu'il faut poursuivre l'utilisation de feu de végétation pour contrôler l'espèce dans les endroits tels que les parcours où elle apparaît effectivement comme adventices. Par ailleurs, il est utile d'exploiter son potentiel agronomique dans le but d'utiliser ces aspects bénéfiques pour le bien-être de la population rurale. Les résultats obtenus sont importants pour l'amélioration des techniques agropastorales et/ou de restauration, afin de limiter l'impact de $C$. odorata sur de tels milieux.

\section{REFERENCES}

Aboh BA, Houinato M, Oumorou M, Sinsin B. 2008. Capacités envahissantes de deux espèces exotiques, Chromolaena odorata (Asteraceae) et Hyptis suaveolens (Lamiaceae), en relation avec l'exploitation des terres de la région de Bétécoucou (Bénin). Belgium Journal of Botany, 141(2): 113-128.

Agonyissa D, Sinsin B. 1998. Productivité et capacité de charge des pâturages naturels au Bénin. Revue d'Elevage et de Médecine Vétérinaire des Pays Tropicaux, 51: 239-249.

Akobundu IO, Ekeleme FE. 1996. Potentials for Chromolaena odorata (L.) In Fallow Management in West and Central Africa, King RM, Robinson H (eds). Third international workshop. www.ehs.cdu .au/chromolaena/3/third.html. Consulté le 24 octobre 2008.

Akpo LE, Masse D, Grouzis M. 2002. Durée de jachère et valeur pastorale de la végétation herbacée en zone soudanienne au Sénégal. Revue d'Elevage et de Médecine. Vétérinaire des Pays Tropicaux, 55 (4): 275-283.

Bodoni EH. 2003. Interactions élevageenvironnement. Dynamique des paysages 
et évolution des pratiques pastorales dans les fronts pionniers du Sud-Ouest du Burkina-Faso. Thèse de doctorat, Univ. Paul Valery, Montpellier III, 295p + annexes.

Braun-Blanquet J. 1932. Plant Sociology. The Study of Plant Communities. English translated revised and edited by Fuller GD, Conard HS. Hafner Press: New York; 439 p.

Daget P, Poissonet J. 1971. Une méthode d'analyse phytosociologique des prairies. Critères d'application. Annales Agronomiques, 22(1): 5-41.

De Foresta H, Schwartz D.1991. Chromolaena odorata and disturbance of natural succession after shifting cultivation: an example from Mayombe, Congo, Central Africa. BIOTROP, 44: 23-41.

Diallo, MS. 1997. Recherches sur l'évolution de la végétation sous l'effet du pâturage dans l'ouest du Burkina Faso (zone soudanienne). Cas de Bondoukuy, Kassaho et Kourouma, Thèse de troisième cycle, Université de Ouagadougou, Faculté des Sciences et Techniques, $147 \mathrm{p}$.

D'Antonio CM, Vitousek PM. 1992. Biological invasions by exotic grasses, the grass/fire cycle, and global change. Annual Review of Ecology \& Systematics, 23: 63-87.

Foxcroft LC, Richardson DM, Rejmànek M, Pysek P. 2010. Alien plant invasions in tropical and sub-tropical savannas: patterns, processes and prospects. Biological Invasions, 12: 3913-3933.

FAO, ISRIC, AISS. 1999. Base de référence mondiale pour les ressources en sols. Rapport sur les resources en sols du monde, $96 \mathrm{p}$.
Henderson L. 2007. Invasive, naturalized and casual alien plants in southern Africa: a summary based on the Southern African Plant Invaders Atlas (SAPIA). Bothalia, 37: 215-248.

Igué AM. 2000. The use of a soil and terrain database for land evaluation procedures Case study of central Benin. PhD thesis, Hohenheimer Bodenkundliche Hefte NR 58, Inst. Für Bodenkunde Uni. Hohenheim.

Oumorou M, Aboh BA, Babatounde S, Houinato M, Sinsin B. 2010. Valeur pastorale, productivité et connaissances endogènes de l'effet de l'invasion, par Hyptis suaveolens L. Poit., des pâturages naturels en Zone soudano-guinéenne (Bénin). International Journal of Biological and Chemical Sciences, 4(4): 1262-1277.

Pagot J. 1985. L'Elevage en Pays Tropicaux, Maisonnoeuve GP (ed). ACCT : Paris.

Radosevich SR, Stubbs MM, Ghersa CM. 2003. Plant invasions process and patterns. Weed Science, 5(1): 254-259.

Raunkiaer C. 1934. The Life Forms of Plants and Statistical Plants Geography. Clarendron Press: Oxford; 632 p.

Richardson DM, Pysek P, Rejmánek M, Barbour MG, Panetta FD, West CJ. 2000.Naturalization and invasion of alien plants: concepts and definitions. Diversity and Distributions, 6: 93-107.

Rivière R. 1991. Manuel d'Alimentation des Ruminants Domestiques en Milieu Tropical. IEMVT $\mathrm{n}^{\circ}$ 9, Collection Manuel et Précis d'Elevage: Paris, France.

Seiny-Boukar L. 2003. Les savanes africaines : des espaces, des hommes, des productions agricoles et des défis à relever pour la recherche. Cahiers Agricultures, 12(4): 209-211. 
Sinsin B. 1993. Phytosociologie, écologie, valeur pastorale, productivité et capacité de charge des pâturages naturels du périmètre Nikki-Kalalé au Nord Bénin. Thèse de doctorat, Univ. Lib. de Bruxelles, Belgique, 390p.

Sokpon N. 1995. Recherches écologiques sur la forêt dense semi-décidue de Pobè au Sud-Est du Bénin: Groupements végétaux, structure, régénération et chute de litière. Thèse de doctorat, Uni. Lib. Bruxelles, Belgique, 350p.

Statistica 6.0. 1998. Logiciel statistica. Maison Alfort, Paris Statsoft, France.

IUCN. 2004. Biodiversity loss and species extinction - managing risk in a changing world. Fact sheet. 3rd IUCN World Conservation Congress Bangkok, Thailand, 17-25 November 2004.

Watkinson AR, Ormerod SJ. 2001. Grasslands, grazing and biodiversity: editors' introduction. Journal of Applied Ecology, 38: 233-237.

Witkowski ETF, Wilson M. 2001. Changes in density, biomass, seed production and soil seed banks of the non-native invasive plant, Chromolaena odorata, along a 15 year chronosequence. Plant Ecology, 152(1): 13-27. 